\title{
siRNA-mediated downregulation of GluN2B in the rostral anterior cingulate cortex attenuates mechanical allodynia and thermal hyperalgesia in a rat model of pain associated with bone cancer
}

\author{
YONGGUANG XU ${ }^{1 *}$, GONGMING WANG $^{1 *}$, XULI ZOU $^{1}$, ZAIQI YANG $^{2}$, \\ QIN WANG ${ }^{3}, \mathrm{HAO} \mathrm{FENG}^{4}$ and MENGYUAN ZHANG ${ }^{1}$ \\ ${ }^{1}$ Department of Anesthesiology, Shandong Provincial Hospital Affiliated to Shandong University, Jinan, Shandong 250021; \\ ${ }^{2}$ Department of Anesthesiology, Taian Central Hospital, Taian, Shandong 270000; ${ }^{3}$ Department of Anesthesiology, \\ Jinan Central Hospital, Jinan, Shandong 250000; ${ }^{4}$ Department of Anesthesiology, \\ The Second Hospital of Shandong University, Jinan, Shandong 250033, P.R. China
}

Received September 12, 2014; Accepted September 15, 2015

DOI: $10.3892 /$ etm.2015.2859

\begin{abstract}
It has previously been suggested that the upregulation of GluN2B-containing N-methyl D-aspartate receptors $(\mathrm{GluN} 2 \mathrm{~B})$ within the rostral anterior cingulate cortex (rACC) may contribute to the development of chronic pain. The present study used a rat model of bone cancer pain in order to investigate whether lentiviral-mediated delivery of small interfering RNAs targeting GluN2B (LV-GluN2B) could attenuate pain associated with bone cancer, by selectively decreasing GluN2B expression within the rACC. Sprague Dawley rats were inoculated with osteosarcoma cells into the intramedullary space of the right tibia in order to induce persistent bone cancer-associated pain. Intra-rACC administration of the lentiviral siRNA was performed in the tumor bearing rats; and reverse transcription-quantitative polymerase chain reaction and western blotting were performed in order to detect the expression levels of GluN2B. Pain behavior changes were evaluated via paw withdrawal threshold and latency determinations. Marked and region-selective decreases in the mRNA and protein expression levels of GluN2B were detected in the rACC following the intra-rACC administration of LV-GluN2B. Furthermore, the rats also exhibited pain behavior changes corresponding to the decreased levels of GluN2B. By post-operative day 14, inoculation of osteosarcoma cells had significantly enhanced mechanical allodynia
\end{abstract}

Correspondence to: Dr Mengyuan Zhang, Department of Anesthesiology, Shandong Provincial Hospital Affiliated to Shandong University, 324 Jingwu Road, Jinan, Shandong 250021, P.R. China

E-mail: mengyuanzhang@fudedu.com

*Contributed equally

Key words: bone cancer pain, rostral anterior cingulate cortex, GluN2B, siRNA, mechanical allodynia and thermal hyperalgesia in the rats, which were subsequently attenuated by the intra-rACC administration of LV-GluN2B. Notably, the paw withdrawal threshold and latency of the tumor-bearing rats had recovered to normal levels, by day 14 post-administration. The results of the present study suggest that GluN2B within the rACC may be a potential target for RNA interference therapy for the treatment of pain associated with bone cancer. Furthermore, the lentiviral vector delivery strategy may be a promising novel approach for the treatment of bone cancer pain.

\section{Introduction}

Occurring in $~ 90 \%$ of patients during their illness (1), pain is one of the most common symptoms associated with cancer. Cancer pain is a severe clinical problem that oncologists continue to encounter, for which the most common current treatment is analgesics, which are often insufficient or addictive. The neurotransmitter system in the brain is an effective regulator of pain perception (2), and the majority of previous studies associated with pain transmission and modulation have focused on the dorsal horn of the spinal cord $(3,4)$. Other studies have demonstrated that neurons in the forebrain, including neurons in the rostral anterior cingulate cortex (rACC), are associated with chronic pain and pain-related perception $(5,6)$. In a mouse model of neuropathic pain, a previous study demonstrated that peripheral nerve injury may trigger both long-term presynaptic and postsynaptic enhancement of excitatory synaptic transmission in layer II/III neurons within the rACC (7). Furthermore, long-term increases in the synaptic insertion of glutamate receptor (GluR)1 subunits in rACC neurons may be induced by peripheral inflammation, which subsequently increases synaptic transmission during chronic pain (8). These results suggest that long-term changes in the plasticity of rACC neurons may have important roles in the development and maintenance of bone cancer-associated pain.

One major feature of long-term plasticity changes in rACC neurons is that it requires the activation of N-methyl D-aspartate (NMDA) receptors. Previous studies have demonstrated that 
chronic pain is selectively enhanced in mice overexpressing GluN2B-containing NMDA receptors (GluN2B) $(9,10)$, suggesting that forebrain GluN2B receptors are critical for the development of chronic pain. Following persistent inflammation, the GluN2B component in NMDA receptor-mediated excitatory postsynaptic currents (EPSCs) was increased due to the increased expression of GluN2B receptors in the rACC (11). Furthermore, the behavioral responses induced by peripheral inflammation during a mechanical allodynia test were inhibited by microinjection into the rACC or systemic administration of GluN2B receptor with selective antagonists (11). These results have been corroborated by genetic studies, in which overexpression of GluN2B in the forebrain of mice selectively enhanced inflammation-related persistent pain, without significantly altering acute pain (12). The antiallodynic effects of GluN2B receptor antagonists have also been demonstrated in other animal models of chronic pain (11). Collectively, these findings suggest that GluN2B receptors within rACC neurons are involved in the development and maintenance of bone cancer-associated pain.

RNA interference (RNAi) is a powerful tool that induces loss-of-function phenotypes by silencing post-transcriptional gene expression $(13,14)$. Long-term knockdown of gene expression has previously been achieved using lentiviral vector constructs that express short hairpin (sh)RNAs in vector-infected cells, including non-dividing cells $(15,16)$. These studies suggest that rACC-specific reductions in GluN2B receptor expression levels may ameliorate debilitating pain, and thus may contribute to the treatment of bone cancer pain. However, to the best of our knowledge, no evidence for relieving the debilitating pain of bone cancer by selectively decreasing GluN2B expression levels in the rACC has yet been reported. In the present study, a rat model of bone cancer-associated pain was used to investigate whether intra-rACC administration of lentiviral-mediated small interfering RNAs targeting GluN2B (LV-GluN2B) successfully decreased the expression levels of GluN2B receptors in the rACC, and if this could relieve debilitating pain. The results demonstrated that rACC-specific reductions in GluN2B receptor expression levels successfully attenuated the debilitating pain associated with bone cancer.

\section{Materials and methods}

GluN2B siRNA sequence design and construction of the siRNA-expressing lentiviral vector. siRNA sequences targeting the GluN2B gene (GenBank: NM_012574) were designed and synthesized as follows by GeneChem Co. (Shanghai, China): Sense, 5'-TCAGAGAGGAATATCCGTAATA-3' and antisense, 5'-TATTACGGATATTCCTCTCTGTTTTTTC-3; and the negative control (NC) siRNA sequences were as follows: Sense, 5'-TCGAGAAAAAACAGAGAGGAATAT CCGTAATA-3' and antisense, 5'-TATTACGGATATTCC TCTCTGA-3. The sequences were subsequently cloned into respective pFU-GW-iRNA expression vectors (GeneChem $\mathrm{Co}$.), containing a green fluorescence protein (GFP) reporter gene and an ampicillin resistance gene.

The lentiviruses were generated as previously described (17) using Lipofectamine ${ }^{\circledR} 2000$ (Invitrogen; Thermo Fisher Scientific Inc., Waltham, MA, USA), a recombinant work vector and package plasmids, which were co-transfected into 293 T cells (Shanghai Bioleaf Biotech Co., Ltd., Shanghai, China). The culture medium (Dulbecco's modified Eagle's medium; Biowest, Nuaillé, France) was collected over 48 h, concentrated, aliquoted, and subsequently stored at $-80^{\circ} \mathrm{C}$ until required. The lentiviral titer was determined by the number of cells expressing GFP multiplied by the corresponding dilutions, using a hole-by-dilution titer assay (18). The final titer of the recombinant virus was $1 \times 10^{9}$ transducing units $/ \mathrm{ml}$. The efficiency of LV-GluN2B in decreasing GluN2B expression levels was examined in vitro by transfecting neurons (data not shown).

Experimental animals. Male Sprague Dawley rats, weighing 220-250 g, were provided by the Experimental Animal Center of Shandong University (Jinan, China) and were maintained in the following identical conditions: A controlled temperature of $22 \pm 1^{\circ} \mathrm{C}$, a $12 \mathrm{~h} \mathrm{light/dark} \mathrm{cycle} \mathrm{and} \mathrm{ad} \mathrm{libitum} \mathrm{access} \mathrm{to}$ food and water. The rats were randomly divided into three groups ( $\mathrm{n}=7 /$ group): Normal saline (NS) group, LV-GluN2B group and LV-NC group. The NS group received no treatment. The LV-GluN2B and LV-NC groups were models of bone cancer pain, the LV-NC group were treated with LV-NC, and the LV-GluN2B group were treated with LV-GluN2B. All experiments were approved by the Committee for Animal Experimentation at Shandong University (Jinan, China) and they complied with the ethical guidelines of the International Association for the Study of Pain.

Cell culture and implantation. Osteosarcoma NCTC 2472 cells, purchased from American Type Culture Collection (ATCC; Manassas, VA, USA) were incubated and subcultured in NCTC 135 medium (Sigma-Aldrich, St. Louis, MO, USA) supplemented with $10 \%$ horse serum (Gibco; Thermo Fisher Scientific, Inc., Waltham, MA, USA) at $37^{\circ} \mathrm{C}$ in a humidified atmosphere containing $5 \% \mathrm{CO}_{2}$ and $95 \%$ air using a Forma ${ }^{\mathrm{TM}}$ incubator (Thermo Fisher Scientific). The cells were passaged twice a week according to ATCC recommendations. A rat model of bone cancer-associated pain was generated as previously described by Schwei et al (19). At day 0, the rats were anesthetized via intraperitoneal injection of $40-50 \mathrm{mg} / \mathrm{kg}$ pentobarbital sodium [1\% in NS], and a superficial incision was made in the right leg in order to expose the tibial plateau. Following this, a 27-gauge needle was used to drill a hole into the medullary cavity, and $10 \mu 1$ phosphate-buffered saline (PBS) supplemented with $2 \times 10^{6}$ osteosarcoma NCTC 2472 cells was subsequently injected using a 29 -gauge needle coupled to a Hamilton syringe. The injection site was closed with tissue glue, thoroughly irrigated with sterile saline in order to prevent the leakage of cells outside the bone, and the wound was closed with skin sutures. Sham-operated controls underwent the same surgical procedure, with the exception that PBS alone was injected into the medullary cavity.

Intra-rACC catheter implantation and administration of viral stocks. The rats were anesthetized via intraperitoneal injection of $40-50 \mathrm{mg} / \mathrm{kg}$ pentobarbital sodium, and were securely placed into a stereotaxic device with the bregma and lambda at a horizontal level. According to the atlas outlined 
by Paxinos et al (20), a 30-gauge stainless steel cannula with a 26-gauge stainless steel stylet plug was bilaterally implanted $0.5 \mathrm{~mm}$ above the rACC injection site (anteroposterior, 2.6; mediolateral, 0.6; and dorsoventral, 2.5 from bregma). The rats recovered and gained weight for 7 days prior to the initiation of the experiments, and three rats presenting with neurological deficits following the surgical procedure were excluded from the study.

Lentiviral stocks were dissolved in NS and over a 3 -min period, a total volume of $0.6 \mu \mathrm{l}$ per hemisphere of NS (vehicle), or lentiviral stocks $\left(1 \times 10^{8}\right.$ PFU) was infused into the rats. The injection syringe was left in place for an additional $1 \mathrm{~min}$ in order to minimize the spread of the drug along the injection track. At the end of the behavioral test, the rats received a $0.6 \mu 1$ infusion of $4 \%$ methylene blue in order to verify the location of the injection site and the extent of the infusion.

Reverse transcription-quantitative polymerase chain reaction $(R T-q P C R)$ analysis. A total of 7 days after vector administration, three different brain regions from each rat: The rACC, the caudal anterior cingulate cortex (cACC), and the ventromedial prefrontal cortex (vmPFC), were harvested using a blunt-end 17-gauge syringe needle (inner diameter, $1 \mathrm{~mm}$ ), according to the atlas outlined by Paxinos et al (20), and snap-frozen in liquid nitrogen until all remaining dissections were collected. Total RNA $(1 \mu \mathrm{g})$ was isolated from the homogenized tissues using TRIzol $^{\circledR}$ (Invitrogen; Thermo Fisher Scientific Inc.), and cDNA was subsequently synthesized using a PrimeScript RT Reagent kit (Takara Bio, Inc.). RT-qPCR was performed on $100 \mathrm{ng} / \mathrm{ml}$ cDNA using a LightCycler ${ }^{\circledR} 480$ system (Roche Diagnostics, Basel, Switzerland) using a SYBR ${ }^{\circledR}$ Premix Ex $\operatorname{Taq}^{T M}$ (Tli RNaseH Plus; Toyobo Co., Ltd., Osaka, Japan) under the following cycle conditions: $95^{\circ} \mathrm{C}$ for $30 \mathrm{sec}, 40$ cycles of $95^{\circ} \mathrm{C}$ for $5 \mathrm{sec}$ and $60^{\circ} \mathrm{C}$ for $30 \mathrm{sec}$, followed by melting at $95^{\circ} \mathrm{C}$ for $5 \mathrm{sec}$ and $60^{\circ} \mathrm{C}$ for $60 \mathrm{sec}$, prior to cooling at $50^{\circ} \mathrm{C}$ for $30 \mathrm{sec}$. The oligonucleotide primers for GluN2B were designed using the NCBI GenBank (NM_012574) as follows: Forward, 5'-TGGCTATCCTGCAGCTGTTTG-3' and reverse, 5'-TGGCTGCTCATCACCTCATTC-3' (Takara Bio, Inc.). $\beta$-actin served as a control for normalization, and the primers were as follows: Forward, 5'-CCCATCTATGAG GGTTACGC-3' and reverse, 5'-TTTAATGTCACGCACGAT TTC-3'. The relative mRNA expression levels were calculated using the $2^{-\Delta \Delta \mathrm{Cq}}$ method (21).

Western blot analysis. Once defined survival times were achieved, the rats were sacrificed via an overdose of $50 \mathrm{mg} / \mathrm{kg}$ pentobarbital,and theirACCtissues weresubsequentlyharvested and maintained at $80^{\circ} \mathrm{C}$. The frozen tissue was homogenized in a lysis solution (10 volumes PBS containing $1 \mathrm{mM} \mathrm{Mg} 2^{+}$, $0.5 \mathrm{mM} \mathrm{Ca}^{2+}, 1 \%$ Triton X-100, $1 \mathrm{mM}$ phenylmethylsulfonyl fluoride). Lysates were centrifuged at $8,000 \mathrm{x} \mathrm{g}$ for $5 \mathrm{~min}$ at $4^{\circ} \mathrm{C}$. Protein concentration was determined using the Bradford method (Shanghai Yapei Biotechnology Co., Ltd., Shanghai, China). The extract samples $(5.0 \mathrm{mg} / \mathrm{ml}, 10 \mu \mathrm{l})$ were separated by $12 \%$ SDS-PAGE, and electroblotted onto polyvinylidene difluoride membranes (Shanghai Resun Biotechnology Co., Ltd., Shanghai, China) using a transfer system (Tanon Science and Technology Co., Ltd., Shanghai, China). Following this, the membranes were blocked with $5 \%$ milk in PBS supplemented with $0.1 \%$ Tween-20 for $1 \mathrm{~h}$ at room temperature and subsequently incubated overnight at $4^{\circ} \mathrm{C}$ with the antibodies against GluN2B (1:400; cat. no. 256) or $\beta$-actin $(1: 1,000$; cat. no. BYK-0061R) (both Shanghai Yua Han Biotechnology Co., Ltd., Shanghai, China), respectively. The blots were then washed and incubated with horseradish peroxidase-conjugated donkey anti-rabbit immunoglobulin G (IgG; 1:1,500; Pierce Biotechnology, Inc., Rockford, IL, USA) for $2 \mathrm{~h}$ at $4^{\circ} \mathrm{C}$, prior to visualization using enhanced chemiluminescence detection reagent (cat. no. 34096; Pierce Biotechnology Inc.). Blots were quantified by measuring and comparing the optical density of the bands using Quantity One version 4.6.2 software (Bio-Rad Laboratories, Inc., Hercules, CA, USA).

GluN2B immunofluorescence. A total of 7 days after vector administration, the rats were anaesthetized with $40-50 \mathrm{mg} / \mathrm{kg}$ pentobarbital prior to the perfusion of $100 \mathrm{ml} \mathrm{NS}$ through the ascending aorta. The rACC tissues were immediately removed and snap-frozen in liquid nitrogen until all the samples were collected. The tissues were subsequently cut into $7 \mu \mathrm{m}$ sections on a cryostat, and all sections were prefixed with acetone, blocked with goat serum at $37^{\circ} \mathrm{C}$ and incubated with mouse anti-GluN2B antibody (cat. no. ab93610; 1:100; Abcam, Cambridge, UK) overnight at $4^{\circ} \mathrm{C}$. Following this, the tissue sections were washed and incubated for $2 \mathrm{~h}$ in tetramethylrhodamine-conjugated Affinipure goat anti-mouse IgG secondary antibody solution (cat. no. SSA004, 1:50, ZSGB-BIO, Beijing China) in a dark room. The stained sections were scanned and images were subsequently captured using an inverted fluorescent microscope (Nikon Corporation, Tokyo, Japan).

\section{Measurements of pain-related behaviors}

Experiment 1: Assessment of pain-related behaviors in tumor-bearing rats. Rats were randomly placed into three groups: Naive, sham and tumor-bearing; and eight rats were subsequently selected at random from each group and tested for pain-related behaviors during a 2-week period: Pre-operative day 0 and on days 3, 5, 7, 10, 14, 21 and 28 following the operation. Mechanical allodynia was assessed in the rats according to the method described by Chaplan et al (22), with minor modifications. The rats were placed in individual transparent plexiglass cages on a metal mesh floor and the threshold of mechanical allodynia was measured using a set of von Frey filaments applied to the right hind paw of each rat. Each von Frey filament was applied vertically to the plantar surface with sufficient force for $6 \mathrm{sec}$, while the filament was gently bent. Paw flinching or brisk withdrawal were considered to be positive responses. Using the 'up and-down' method, the lowest von Frey filament that induced $>3$ positive responses was recorded as the paw withdrawal mechanical threshold (PWMT). Thermal hyperalgesia was measured according to the method described by Hargreaves et al (23), with the paw withdrawal thermal latency (PWTL) value recorded in response to radiant heat. Rats were placed in individual transparent plexiglass cages on a $3 \mathrm{~mm}$-thick-glass floor and a radiant thermal stimulator (BME410A; Institute of Biological Medicine, Academy of Medical Science, Huaibei, 
China) was positioned under the glass floor and subsequently focused on the plantar surface of the rat's hind paw. PWTL was defined as the time that elapsed between the onset of the radiant heat and the characteristic lifting or licking of the hind paw. A cut-off time of $25 \mathrm{sec}$ was imposed in order to avoid tissue damage. Each rat completed five trials with 5-min intervals, and the mean PWTL was obtained from the latter three stimuli.

Experiment 2: Effects of intra-rACC administration of LV-GluN2B on pain behaviors induced by bone cancer. Tumor-bearing rats, weighing 220-250 g, were randomly divided into three groups: Normal saline (NS), lentivirus negative control (LV-NC), and LV-GluN2B; which were respectively intra-rACC administered with NS, LV-NC, or LV-GluN2B to a total volume of $0.6 \mu \mathrm{l}$ per hemisphere. Pain-related behaviors were assessed prior to administration, which were regarded as baseline, and at post-administrative days 0, 3, 7, 10 and 14 . The locomotor activity of the rats was measured using perspex boxes $(230 \times 280 \times 210 \mathrm{~mm})$ equipped with two parallel infrared beams, one at each end of the base of the cage.

Statistical analysis. Data are expressed as the mean \pm standard error of the mean. Rats were randomly assigned to the various treatment groups. SPSS 19.0 (IBM SPSS, Armonk, NY, USA) was used to statistically analyze data. Two-way repeated analysis of variance (ANOVA) was used to detect differences in body weight, PWMT and PWTL among the groups; whereas one-way ANOVA with Student-Newman-Keuls post hoc analysis was used to detect differences in the numbers of GluN2B-immune-positive cells, and the mRNA and protein expression levels of GluN2B among the groups. $\mathrm{P}<0.05$ was considered to indicate a statistically significant difference.

\section{Results}

Tumor-bearing rats exhibit increased mechanical allodynia and thermal hyperalgesia. In order to induce ongoing bone cancer pain behaviors in the rat model, osteosarcoma cells were inoculated into the intramedullary space of the right tibia. No significant differences in body weight gain were demonstrated among the naive, sham-operated, and tumor-bearing groups (Fig. 1A, P>0.05) in the time period examined. Tumor-bearing rats demonstrated increased mechanical allodynia and thermal hyperalgesia from postoperative days 10-28, as compared with the naive and sham-operated groups. A progressive decrease of PWMT on the ipsilateral side of the tumor-bearing rats was demonstrated on postoperative day $10(9.2 \pm 1.4 \mathrm{~g})$, which was significantly less than the threshold observed in the naive $(11.8 \pm 2.1 \mathrm{~g})$ or sham-operated rats $(12.1 \pm 2.4 \mathrm{~g})$, and this persisted for the remainder of the study (Fig. 1B). Furthermore, tumor-bearing rats demonstrated a reduction in PWTL on the ipsilateral side at postoperative day 10 , which gradually decreased along with the development of bone cancer-associated pain (Fig. 1C).

Specificity and efficiency of GluN2B deletions within the $r A C C$ following intra-rACC administration of $L V-G l u N 2 B$. In order to investigate whether LV-GluN2B selectively decreased the expression levels of GluN2B within the rACC,
intra-rACC administration of lentiviral RNAs was performed in tumor-bearing rats. Detection of GluN2B mRNA expression levels in the rats demonstrated that GluN2B knockdown was selectively limited to the $\mathrm{rACC}$, and was statistically significant $(\mathrm{P}<0.05)$. Fig 2 demonstrates the decrease in GluN2B expression levels within the rACC following LV-GluN2B infection. In the NS, LV-GluN2B and LV-NC groups, the relative mRNA expression levels of GluN2B within the rACC were 100, 42 and 98, respectively (Fig. 2A). Since GluN2B is also abundantly distributed in other forebrain areas, the mRNA expression levels of GluN2B in the cACC and vmPFC were also examined, and no difference in expression was detected (Fig. 2B and C).

Intra-rACC injection of $L V$-GluN2B specifically decreases GluN2B protein expression levels in the $r A C C$. The effects of intra-rACC delivery of LV-GluN2B on the protein expression levels of GluN2B in rACC neurons were also analyzed via immunofluorescence staining. As outlined in Fig. 3, following intra-rACC injection of LV-GluN2B, the expression of GluN2B protein in the rACC neurons decreased. Furthermore, the number of GluN2B-positive cells in the $\mathrm{ACC}$ were significantly decreased in rats treated with LV-GluN2B, as compared with that of the NS or LV-GluN2B controls $(82 \pm 16$ vs. $146 \pm 22$ or 138 \pm 20 ; Fig. $3 \mathrm{~A}$ and $\mathrm{B} ; \mathrm{P}<0.05)$.

Western blot analysis was subsequently performed in order to compare the expression levels of the GluN2B subunits in the rACC among the groups. Consistent with the immunohistochemical results, intra-rACC injection of LV-GluN2B resulted in a $49.6 \%$ reduction in GluN2B protein expression levels, as compared with the LV-NC and blank control groups $(\mathrm{P}<0.05)$ (Fig. 4A and $\mathrm{B})$.

Intra-rACC injection of $\mathrm{LV}$-GluN2B reduces tactile allodynia and thermal hyperalgesia in a model of bone cancer-associated pain, without altering locomotor activity. To investigate whether the downregulation of LV-GluN2B in the rACC could attenuate bone cancer-associated pain, the pain-related behaviors of the rats were observed for 14 days following the injection of the lentiviral vectors. The rats exhibited a normal appearance, level of activity and regular appetite following intra-rACC injection of the lentiviral vectors. Furthermore, no significant differences in body weight were demonstrated among the sham, NS, LV-NC or LV-GluN2B groups. Notably, all tumor-bearing rats demonstrated increased mechanical allodynia and thermal hyperalgesia at day 10 following osteosarcoma inoculation; whereas intra-rACC injection of LV-GluN2B improved tactile allodynia in the tumor-bearing rats by day 3. Following administration, the PWMT of the ipsilateral side of the tumor-bearing rats significantly increased from the baseline $(5.50 \pm 1.4 \mathrm{~g})$ to $8.3 \pm 2.8 \mathrm{~g}(\mathrm{P}<0.05)$, and gradually recovered to $10.5 \pm 3.2 \mathrm{~g}$ by day 7 and baseline by day 14 (Fig. 5A). Similarly, the PWTL of the tumor-bearing rats recovered to $10.3 \pm 2.6 \mathrm{sec}$ by post-administrative day 7, and had returned to the baseline by day 14 (Fig. 5B). Conversely, intra-rACC administration of NS or LV-NC had no notable effect on tactile allodynia or thermal hyperalgesia in the tumor-bearing rats (Fig. 5).

To examine the effects of intra-rACC injection of LV-GluN2B on the locomotion of the rats, baseline locomotion was measured as the total number of beam breaks in $2 \mathrm{~h}$ using perspex boxes $(230 \times 280 \times 210 \mathrm{~mm})$ equipped with two parallel 
A

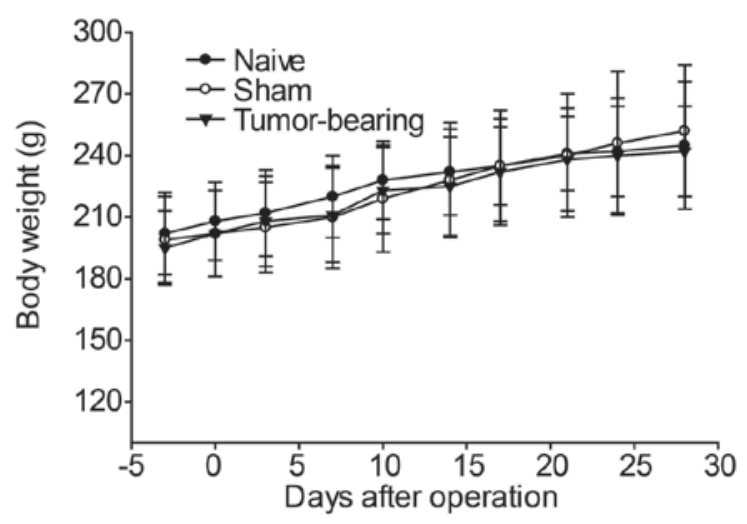

B

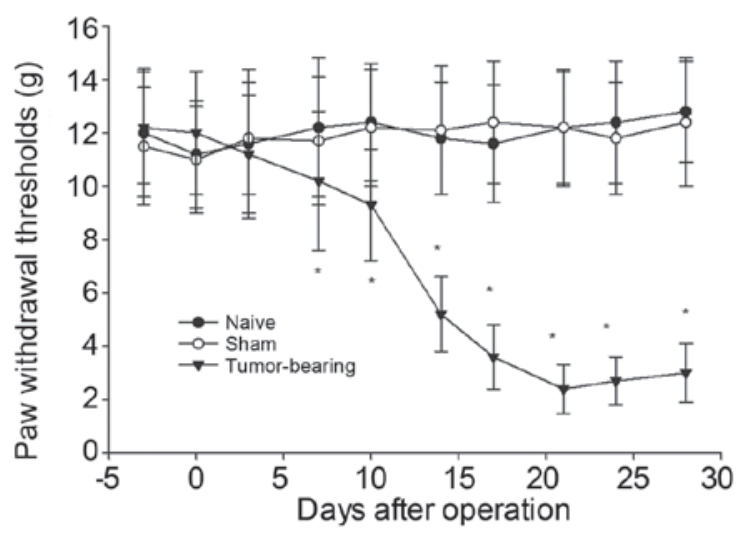

C

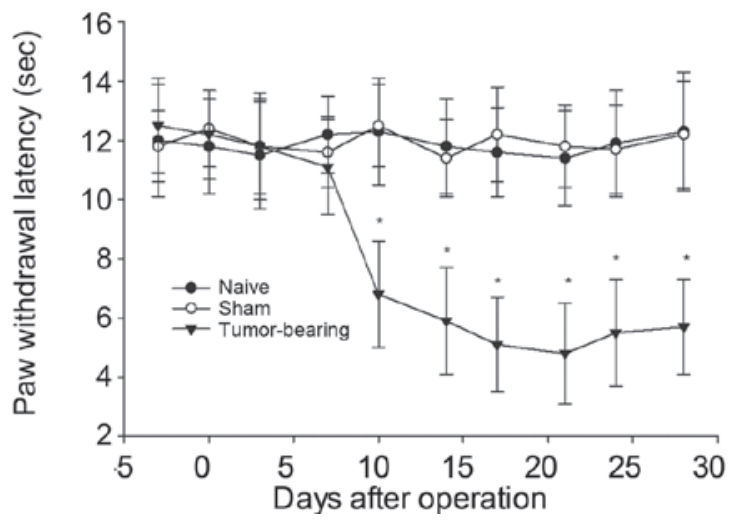

Figure 1. Changes in body weight and responsiveness to mechanical or thermal stimuli in naive, sham-operated and tumor-bearing rats over time (A) No significant differences in body weight gain were demonstrated among the naive, sham-operated and tumor-bearing groups over the time period examined. (B) The withdrawal thresholds in response to non-noxious mechanical stimulation by von Frey filaments were significantly decreased in the ipsilateral hind paws of tumor-bearing rats by postoperative day 10 . (C) Tumor-bearing rats demonstrated a decrease in paw withdrawal therma latency on the ipsilateral side at postoperative day 10, which then decreased gradually with the development of the cancer pain. Data are expressed as the mean \pm standard error of the mean. ${ }^{*} \mathrm{P}<0.05$ vs. the sham-operated group.

infrared beam arrays. No difference in baseline locomotor activity was determined among the NS, LV-NC and LV-GluN2B groups (Fig. 6).

\section{Discussion}

The present study demonstrated that GluN2B within the rACC may be a potential RNAi target for the treatment of pain associated with bone cancer. Furthermore, intra-rACC delivery of
A

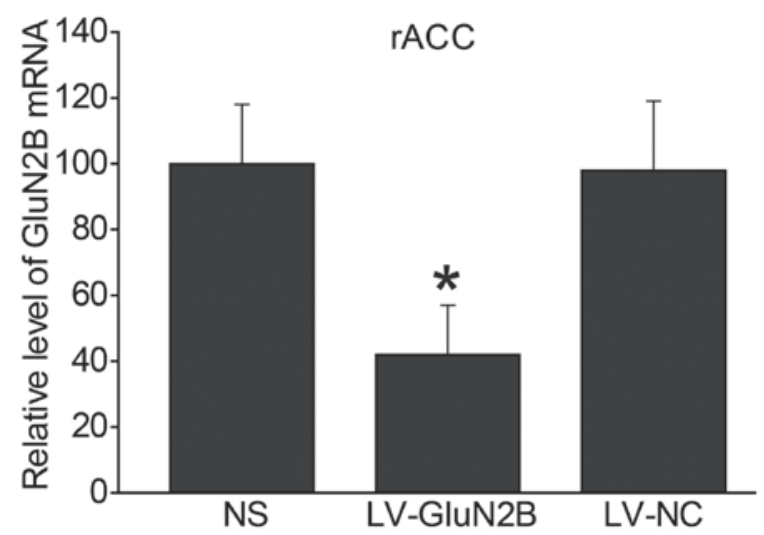

B

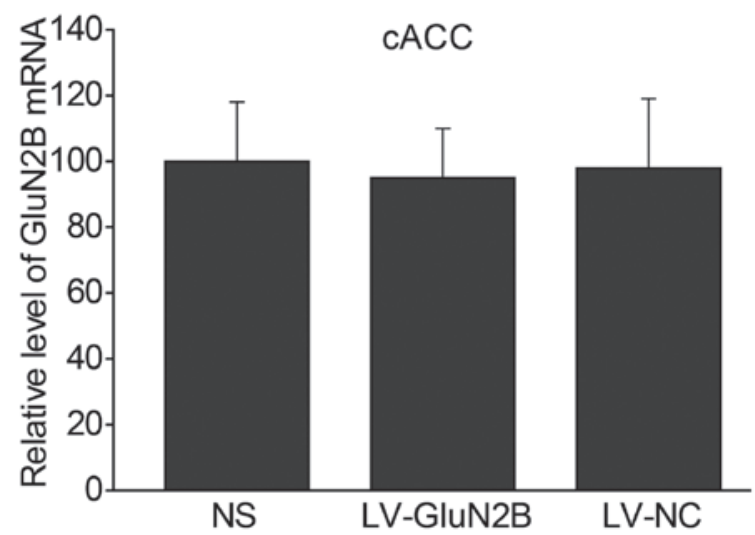

C

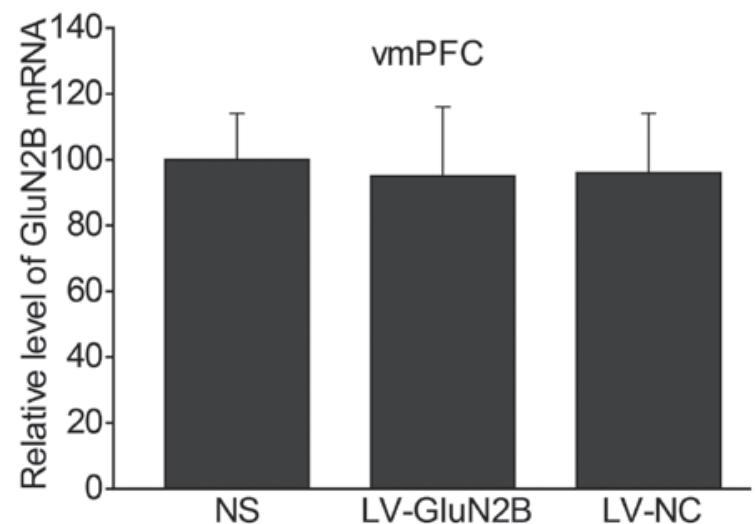

Figure 2. GluN2B mRNA expression levels decreased within the rACC following LV-GluN2B injection. Reverse transcription-quantiative polymerase chain reaction analysis of GluN2B mRNA expression levels in the (A) rACC, (B) vmPFC and (C) cACC following LV-GluN2B injection in the rACC ( $n=5-6$ per group). No changes were detected in the mRNA expression levels of GluN2B in the cACC or vmPFC following LV-GluN2B injection. Data are presented as the mean \pm standard error of the mean. ${ }^{*} \mathrm{P}<0.05$ vs. the LV-NC group. GluN2B, GluN2B-containing NMDA receptors; rACC, rostral anterior cingulate cortex; cACC, caudal anterior cingulate cortex; vmPFC, ventromedial prefrontal cortex; NS, normal saline; LV-NC, lentiviral negative control; LV-GluN2B, lentiviral-mediated delivery of small interfering RNAs targeting GluN2B.

lentiviral vectors was effective at silencing GluN2B in rACC neurons and providing a considerable antinociceptive therapeutic effect for bone cancer-associated pain.

Previous animal models of cancer pain have been developed in order to examine the mechanisms that underlie tumor-evoked pain and hyperalgesia. Using models in which osteolytic fibrosarcoma cells are implanted into the 
A

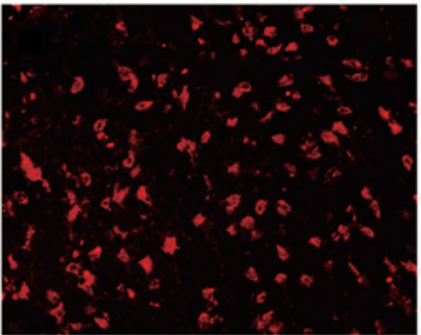

NS

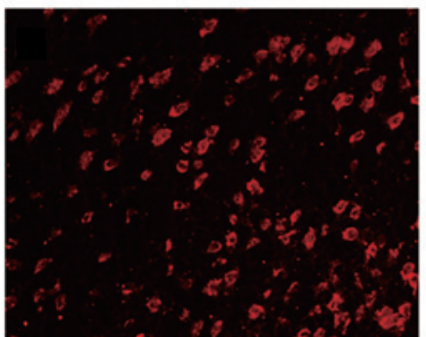

LV-NC

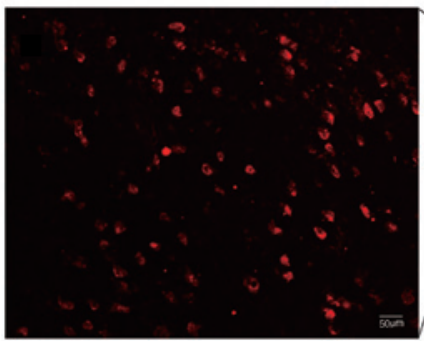

LV-GluN2B

B

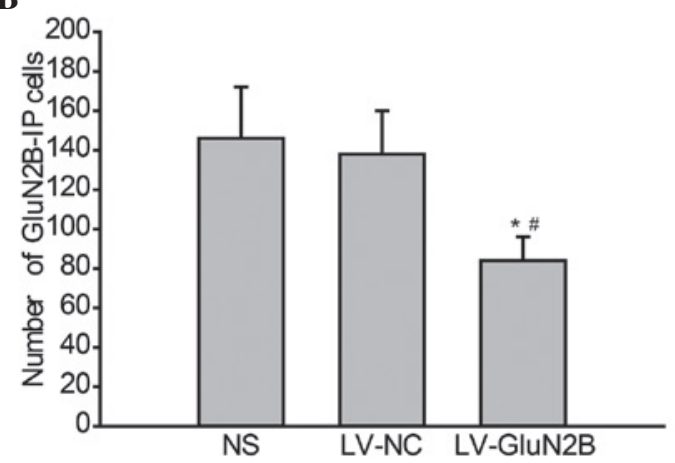

Figure 3. Immunofluorescence staining of GluN2B protein in neurons rACC. (A) Photomicrographs demonstrating protein expression of GluN2B in the rACC neurons at day 7 following intra-rACC injection of LV-GluN2B. (B) Quantification of the GluN2B-IP cells demonstrated that intra-rACC delivery of LV-GluN2B significantly decreased GluN2B protein expression levels in the rACC neurons, as compared with the NS or LV-NC groups. ${ }^{*} \mathrm{P}<0.05$ vs. NS; ${ }^{*} \mathrm{P}<0.05$ vs. LV-NC control. rACC, rostral anterior cingulate cortex; GluN2B, GluN2B-containing N-methyl D-aspartate receptors; NS, normal saline; LV-NC, lentivirus negative control; LV-GluN2B, lentiviral-mediated delivery of small interfering RNAs targeting GluN2B; IP, immuno-positive.

A NS LV-NC

\section{LV-GluN2B}

\section{GluN2B}

$\beta$-actin

$\mathbf{B}$

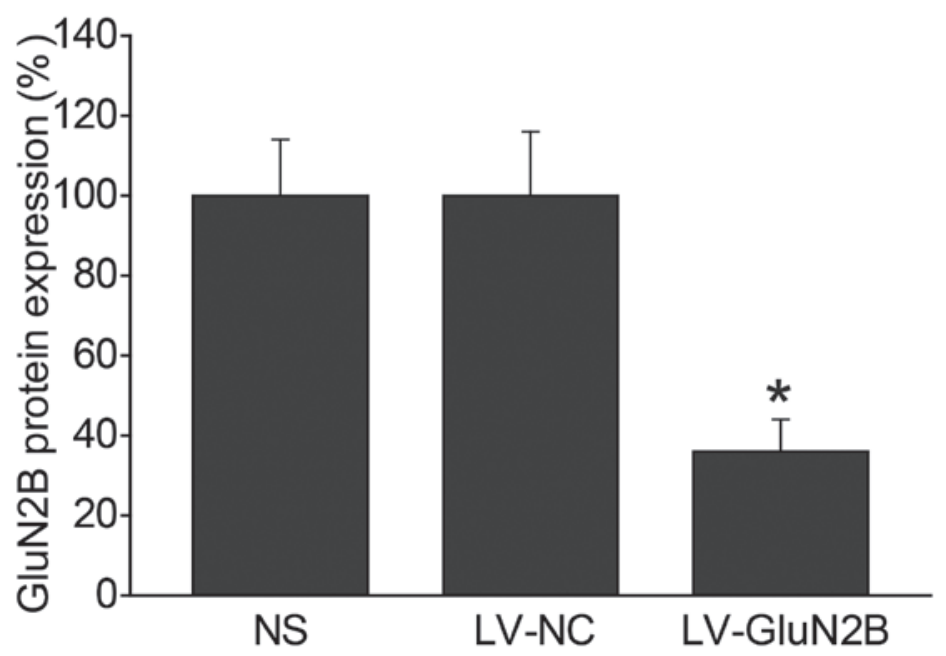

Figure 4. Attenuation of the protein expression levels of GluN2B in neurons of the rACC by intra-rACC administration of LV-GluN2B. (A) Western blot of GluN2B protein expression in the rACC neurons after intra-rACC delivery of NS, LV-NC or LV-GluN2B. (B) Western blot analysis demonstrated that GluN2B protein was significantly downregulated (49.6\%) at day 7 following intra-rACC delivery of LV-GluN2B. ${ }^{*} \mathrm{P}<0.05$ vs. the LV-NC or NS groups ( $\mathrm{n}=4$ ). GluN2B, GluN2B-containing N-methyl D-aspartate receptors; rACC, rostral anterior cingulate cortex; NS, normal saline; LV-NC, lentiviral negative control; LV-GluN2B, lentiviral-mediated delivery of small interfering RNAs targeting GluN2B.

humerus, femur, tibia, or calcaneus bone, researchers have begun to elucidate the pathophysiological processes by which cancer produces pain (24), and evaluate novel approaches for the treatment of cancer pain (19). In the present study, 
A

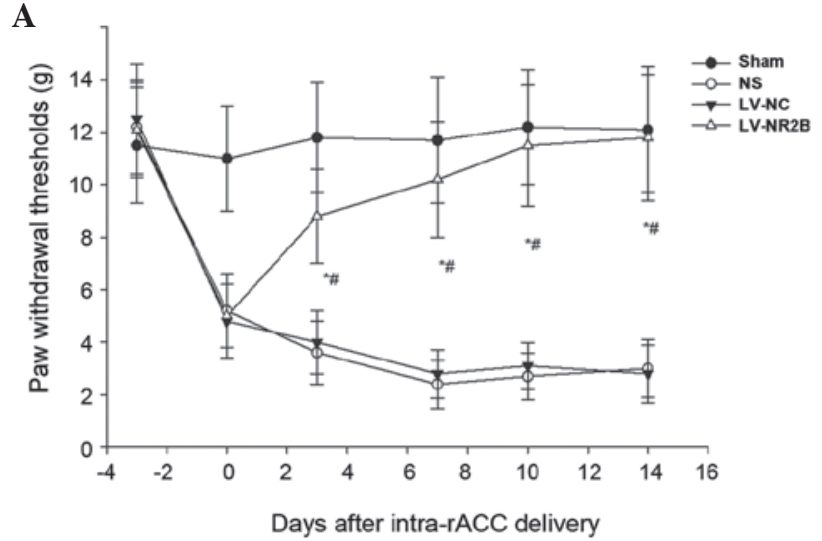

B

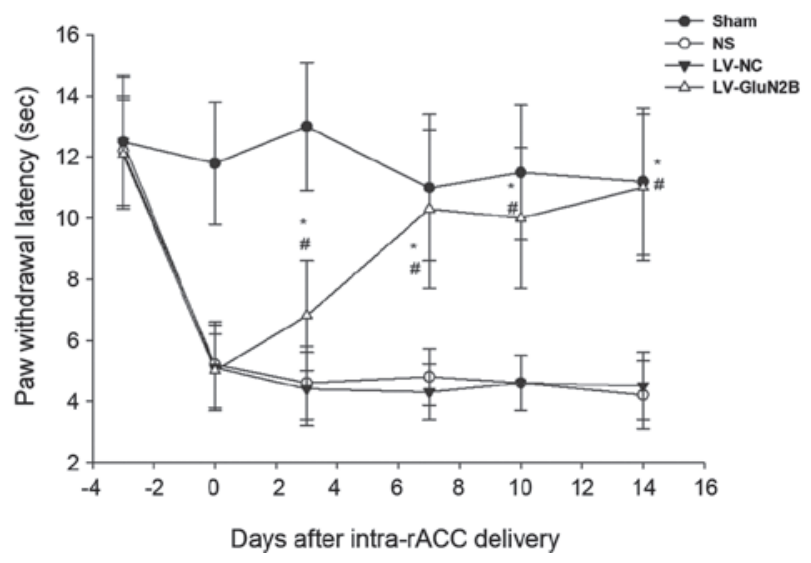

Figure 5. Effects of intra-rACC administration of LV-GluN2B on tactile allodynia and thermal hyperalgesia in tumor-bearing rats. (A) Tumor-bearing rats treated with LV-GluN2B demonstrated a significant increase in the withdrawal threshold to non-noxious mechanical stimulation by von Frey filament, as compared with the NS or LV-NC groups. (B) The decreased withdrawal latency to thermal stimulation in the rats was reversed by treatment with $L V-G l u N 2 B$ injection. " $\mathrm{P}<0.05$ vs. the NS group; ${ }^{~} \mathrm{P}<0.05$ vs. the LV-NC group at each time point. rACC, rostral anterior cingulate cortex; LV-GluN2B, lentiviral-mediated delivery of small interfering RNAs targeting GluN2B; NS, normal saline; LV-NC, lentiviral negative control.

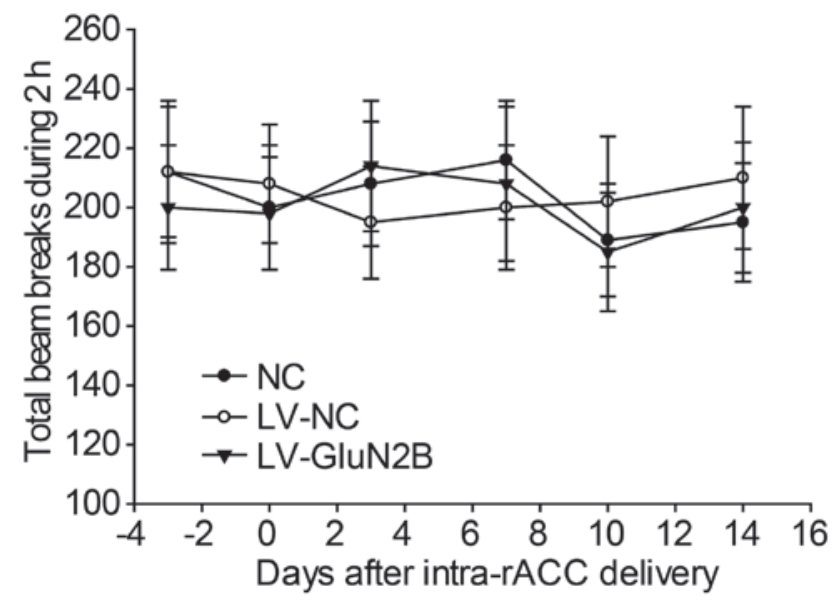

Figure 6. Effects of intra-rACC injection of LV-GluN2B on the locomotor activity of rats. Rats were habituated to individual cages for at least $1 \mathrm{~h}$ prior to the initiation of measurements. Data are expressed as the total number of beam breaks (the mean \pm standard error of the mean) recorded over $2 \mathrm{~h}$. No significant differences were demonstrated among these groups during the 14-day monitoring period. rACC, rostral anterior cingulate cortex; $\mathrm{NC}$, normal control; LV-NC, lentiviral negative control; LV-GluN2B, lentiviral-mediated delivery of small interfering RNAs targeting GluN2B. a rat model of bone cancer-associated pain was created by injecting osteosarcoma cells into the intramedullary space of the femur. The rats that received the osteosarcoma injection exhibited mechanical allodynia and thermal hyperalgesia by post-operative day 10; whereas those receiving the sham injection exhibited no detectable pain-related behavior throughout the observation period. These pain-related behaviors remained unchanged, as assessed on post-operative days 14, 21 and 28. Therefore, these results suggested that the rat bone cancer pain model used in the present study may be pathologically and physiologically applicable to the intended clinical situation.

RNAi-mediated gene silencing is generated through the expression of a vector-mediated RNAi anywhere in the genome (25), and this technique has been tested in clinical trials as a potential novel therapy for various diseases (26). Viral delivery of shRNA expression cassettes allows efficient transduction in tissues such as the brain and liver. For example, recombinant adeno-associated viral vectors expressing shRNA have been used in a previous study to knockdown a target gene associated with Huntington's disease (27) and Anesti et al (28) demonstrated that herpes simplex virus may be used to efficiently deliver RNAi into peripheral neurons in vivo. Therefore, viral-based vectors have been developed as an alternative therapeutic strategy. Lentiviral vectors were selected as a gene delivery tool in the present study due to their relatively large cloning capacity and their ability to induce limited inflammation, express shRNA, and induce stable and long-term gene silencing in both dividing and non-dividing cells. In the present study, siRNA sequences targeting the GluN2B gene were cloned into a pFU-GW-iRNA lentiviral vector, and the recombinant lentiviral vectors were subsequently injected into the rACC of rats. Following intra-rACC administration of LV-GluN2B, RT-qPCR and western blot analysis demonstrated a marked decrease in the mRNA and protein expression levels of GluN2B in the rACC. Furthermore, quantitative analyses of the mRNA expression levels following the injection of LV-GluN2B into rACC revealed that the reduced GluN2B expression levels demonstrated in the present study were statistically significant and largely limited to the rACC. These results indicated that the lentiviral vector delivery strategy employed in the present study may be a promising novel approach for the treatment of bone cancer-associated pain.

In the present study, the specific knockdown of GluN2B receptors in the rACC successfully alleviated the tactile allodynia and thermal hyperalgesia induced in the rat model of bone cancer-associated pain. These results corroborate a previous study, which demonstrated that microinjection of a selective metabotropic GluR agonist, tACPD, into the rACC produced facilitation of the tail-flick reflex (29). It has been demonstrated that spinal nociception is subject to descending modulation from supraspinal structures, including neurons in the ACC and insular cortex. The ACC forms part of the affective pain response system and contributes to various cortical functions, including the perception of pain and the learning processes associated with the prediction or avoidance of noxious stimuli (30). A previous study has demonstrated that neurons in the ACC respond to acute noxious stimuli or nerve 
injury (31), and lesions in the ACC have been demonstrated to affect the behavioral response of rats in the hot-plate test (4). Furthermore, it has previously been reported that efferents from the ACC innervate the periaqueductal gray (30). A previous study using afferent vagal stimulation or stimulation in the rostral ventromedial medulla demonstrated that the latency for facilitatory modulation of the spinal sensory neurons is longer than that for inhibition, and the involvement of the rostral loop has been suggested (32). These findings suggested that neuronal activity in the ACC may affect spinal nociception through descending modulatory systems.

The results of the present study suggested that GluN2B within the rACC is a potential RNAi target for the treatment of pain associated with bone cancer. A previous study demonstrated that, in mice genetically engineered to overexpress GluN2B, chronic pain was selectively enhanced, whereas acute or physiological pain were unaffected (12); providing the first genetic evidence that NMDA GluN2B receptors in the forebrain are associated with chronic pain. In another previous study using a complete Freund's adjuvant-induced animal model for chronic inflammation, the expression levels of GluN2B receptors in the ACC were increased over a long period of time following persistent inflammation, thus increasing the GluN2B component in NMDA receptor-mediated EPSCs (12). Furthermore, a previous study utilized the behavioral allodynia test and demonstrated that microinjection into the ACC or systemic administration of GluN2B receptor with selective antagonists inhibited behavioral responses to peripheral inflammation (11). These results are consistent with genetic studies, which have demonstrated that mice with GluN2B forebrain overexpression selectively enhanced inflammation-related persistent pain without significant changes in acute pain. The antiallodynic effects of NMDA GluN2B receptor antagonists have also been reported in other animal models of chronic pain (33). These results indicated that the development and maintenance of bone cancer-associated pain is associated with the GluN2B subunit of NMDA receptors in the rACC.

In addition to the rACC, GluN2B is abundantly distributed in other areas of the forebrain. Therefore, the locomotion of the rats was observed in order to determine whether intra-rACC injection of LV-GluN2B produced any notable effects on motor activity. No significant differences were determined among the groups during the 14-day monitoring period. Subsequent RT-qPCR and western blot analysis demonstrated that intra-injection of LV-GluN2B specifically decreased GluN2B expression levels within the rACC and had no obvious effects on the other areas of the forebrain. These results further support the hypothesis that RNAi-mediated gene silencing is a potential therapeutic tool for the treatment of various nervous diseases.

In conclusion, the present study demonstrated that cancer pain-related behaviors may be ameliorated by intra-rACC administration of LV-GluN2B and the effects of LV-GluN2B may be associated with a reduction in the expression of GluN2B in rACC neurons. Furthermore, the present study may help elucidate the central mechanism of bone cancer pain and provide a novel therapeutic strategy for the prevention and/or treatment of bone cancer pain by focusing on the plasticity alterations occurring at the ACC synapses.

\section{Acknowledgements}

The present study was supported by the National Science Council of China (grant no. 30872433) and the Science and Technology Foundation of Shandong Province (grant no. 2010GWZ2023P).

\section{References}

1. Peng WL, Wu GJ, Sun WZ, Chen JC and Huang AT: Multidisciplinary management of cancer pain: A longitudinal retrospective study on a cohort of end-stage cancer patients. J Pain Symptom Manage 32: 444-452, 2006.

2. Kuner R: Central mechanisms of pathological pain. Nat Med 16: 1258-1266, 2010.

3. Price DD: Psychological and neural mechanisms of the affective dimension of pain. Science 288: 1769-1772, 2000.

4. Pastoriza LN, Morrow TJ and Casey KL: Medial frontal cortex lesions selectively attenuate the hot plate response: Possible nocifensive apraxia in the rat. Pain 64: 11-17, 1996.

5. Vogt BA: Pain and emotion interactions in subregions of the cingulate gyrus. Nat Rev Neurosci 6: 533-544, 2005.

6. Zhuo M: Cortical excitation and chronic pain. Trends Neurosci 31: 199-207, 2008.

7. Xu H, Wu LJ, Wang H, Zhang X, Vadakkan KI, Kim SS, Steenland HW and Zhuo M: Presynaptic and postsynaptic amplifications of neuropathic pain in the anterior cingulate cortex. J Neurosci 28: 7445-7453, 2008.

8. Bie B, Brown DL and Naguib M: Increased synaptic GluR1 subunits in the anterior cingulate cortex of rats with peripheral inflammation. Eur J Pharmacol 653: 26-31, 2011.

9. Wei F, Qiu CS, Kim SJ, Muglia L, Maas JW, Pineda VV, Xu HM, Chen ZF, Storm DR, Muglia LJ and Zhuo M: Genetic elimination of behavioral sensitization in mice lacking calmodulin-stimulated adenylyl cyclases. Neuron 36: 713-726, 2002.

10. Tang YP, Shimizu E, Dube GR, Rampon C, Kerchner GA, Zhuo M, Liu G and Tsien JZ: Genetic enhancement of learning and memory in mice. Nature 401: 63-69, 1999.

11. Wu LJ, Toyoda H, Zhao MG, Lee YS, Tang J, Ko SW, Jia YH, Shum FW, Zerbinatti CV, Bu G, et al: Upregulation of forebrain NMDA NR2B receptors contributes to behavioral sensitization after inflammation. J Neurosci 25: 11107-111016, 2005.

12. Wei F, Wang GD, Kerchner GA, Kim SJ, Xu HM, Chen ZF and Zhuo M: Genetic enhancement of inflammatory pain by forebrain NR2B overexpression. Nat Neurosci 4: 164-169, 2001.

13. Fire $\mathrm{A}, \mathrm{Xu} \mathrm{S}$, Montgomery MK, Kostas SA, Driver SE and Mello CC: Potent and specific genetic interference by double-stranded RNA in Caenorhabditis elegans. Nature 391: 806-811, 1998.

14. Dorn G, Patel S, Wotherspoon G, Hemmings-Mieszczak M, Barclay J, Natt FJ, Martin P, Bevan S, Fox A, Ganju P, et al: siRNA relieves chronic neuropathic pain. Nucleic Acids Res 32: e49, 2004.

15. Naldini L, Blömer U, Gallay P, Ory D, Mulligan R, Gage FH, Verma IM and Trono D: In vivo gene delivery and stable transduction of nondividing cells by a lentiviral vector. Science 272: 263-267, 1996.

16. Manjunath N, Wu H, Subramanya S and Shankar P: Lentiviral delivery of short hairpin RNAs. Adv Drug Deliv Rev 61: 732-745, 2009.

17. Coleman JE, Huentelman MJ, Kasparov S, Metcalfe BL, Paton JF, Katovich MJ, Semple-Rowland SL and Raizada MK: Efficient large-scale production and concentration of HIV-1-based lentiviral vectors for use in vivo. Physiol Genomics 12: 221-228, 2003.

18. Déglon N, Tseng JL, Bensadoun JC, Zurn AD, Arsenijevic Y, Pereira de Almeida L, Zufferey R, Trono D and Aebischer P: Self-inactivating lentiviral vectors with enhanced transgene expression as potential gene transfer system in Parkinson's disease. Hum Gene Ther 11: 179-190, 2000.

19. Schwei MJ, Honore P, Rogers SD, Salak-Johnson JL, Finke MP, Ramnaraine ML, Clohisy DR and Mantyh PW: Neurochemical and cellular reorganization of the spinal cord in a murine model of bone cancer pain. J Neurosci 19: 10886-10897, 1999.

20. Paxinos G, Watson CR and Emson PC: AChE-stained horizontal sections of the rat brain in stereotaxic coordinates. J Neurosci Methods 3: 129-149, 1980. 
21. Livak KJ and Schmittgen TD: Aanlysis of relative gene expression data using real-time quantitative PCR and 2(-Delta Delta C(T)) Method. Methods 25: 402-408, 2001.

22. Chaplan SR, Bach FW, Pogrel JW, Chung JM and Yaksh TL: Quantitative assessment of tactile allodynia in the rat paw. J Neurosci Methods 53: 55-63, 1994.

23. Hargreaves K, Dubner R, Brown F, Flores C and Joris J: A new and sensitive method for measuring thermal nociception in cutaneous hyperalgesia. Pain 32: 77-88, 1988.

24. Wacnik PW, Eikmeier LJ, Ruggles TR, Ramnaraine ML, Walcheck BK, Beitz AJ and Wilcox GL: Functional interactions between tumor and peripheral nerve: Morphology, algogen identification, and behavioral characterization of a new murine model of cancer pain. J Neurosci 21: 9355-9366, 2001.

25. Zhai Z, Sooksa-nguan $\mathrm{T}$ and Vatamaniuk OK: Establishing RNA interference as a reverse-genetic approach for gene functional analysis in protoplasts. Plant Physiol 149: 642-652, 2009.

26. Shrey K, Suchit A, Nishant M and Vibha R: RNA interference: Emerging diagnostics and therapeutics tool. Biochem Biophys Res Commun 386: 273-277, 2009.
27. Franich NR, Fitzsimons HL, Fong DM, Klugmann M, During MJ and Young D: AAV vector-mediated RNAi of mutant huntingtin expression is neuroprotective in a novel genetic rat model of Huntington's disease. Mol Ther 16: 947-956, 2008.

28. Anesti AM, Peeters PJ, Royaux I and Coffin RS: Efficient delivery of RNA interference to peripheral neurons in vivo using herpes simplex virus. Nucleic Acids Res 36: e86, 2008.

29. Calejesan AA, Kim SJ and Zhuo M: Descending facilitatory modulation of a behavioral nociceptive response by stimulation in the adult rat anterior cingulate cortex. Eur J Pain 4: 83-96, 2000.

30. Vogt BA and Gabriel M (eds.): Neurobiology of cingulate cortex and limbic thalamus: A comprehensive handbook. XIII, Birkhäuser, Boston, p639, 1993.

31. Talbot JD, Marrett S, Evans AC, Meyer E, Bushnell MC and Duncan GH: Multiple representations of pain in human cerebral cortex. Science 251: 1355-1358, 1991.

32. Ren K, Randich A and Gebhart GF: Electrical stimulation of cervical vagal afferents. I. central relays for modulation of spinal nociceptive transmission. J Neurophysiol 64: 1098-1114, 1990.

33. Salte K, Lea G, Franek M and Vaculin S: Baclofen reversed thermal place preference in rats with chronic constriction injury. Physiol Res: Oct 8, 2015 (Epub ahead of print). 